\title{
Reduced Osteoglycin Protein Expression Correlates with Lymph Node Metastasis and Low Differentiation in Human Gastric Cancer
}

\section{Chengjia Qian}

Jiangnan University

Changyong Zhao

Jiangnan University

Qizhong Gao

Jiangnan University

Jiming Gu

Jiangnan University

Weifeng Han

Jiangnan University

Hui Jiang

Jiangnan University

Liugen Jin

Jiangnan University

Xiaowei Qi

Jiangnan University

Bojian Fei

Jiangnan University

Huiming Tu ( $\nabla$ thmercp@163.com )

Jiangnan University

\section{Research Article}

Keywords: gastric cancer, Osteoglycin, lymph node metastasis, differentiation, biomarker

Posted Date: February 21 st, 2022

DOI: https://doi.org/10.21203/rs.3.rs-775886/v1

License: (c) (1) This work is licensed under a Creative Commons Attribution 4.0 International License.

Read Full License 


\section{Abstract \\ Background}

Gastric cancer (GC) is a highly malignant disease for which the development of novel biomarkers and therapeutic targets is urgently required. Decreased expression of Osteoglycin (OGN) has been observed in the progression of a variety of human cancers; however, the pathologic significance of OGN in GC remains unexplored. We aimed to detect the expression of OGN in gastric cancer tissues, and to evaluated the relationship between OGN expression and the clinico-pathological features in GC patients.

\section{Methods}

In this study, the expression levels of OGN were detected by immunohistochemistry (IHC) with the cohort including 44 gastric cancer samples and 40 non-tumorous samples. The data was statistically analyzed using GraphPad Prism 8.0.

\section{Results}

We found that OGN expression was downregulated in GC tissues compared to non-tumor counterparts. Furthermore, GC samples with lower OGN levels demonstrated higher lymph node metastasis. Histological analysis further confirmed that OGN downregulation was related to lower differentiation status of GC.

\section{Conclusions}

Collectively, our results indicate that dysregulation of OGN might be involved in GC development and that the expression of OGN may serve as a potential biomarker for monitoring of the progression of GC.

\section{Background}

Gastric cancer (GC) is the fifth most common malignancy globally.[1] It is estimated that approximately one million cases of GC occur each year,[2,3] with more than half of all GC cases emerging from East Asia.[4,5] Despite significant advances in diagnostic techniques, surgical and chemotherapeutic approaches, GC is still the third leading cause of cancer mortality worldwide.[6, 7] The majority of GC cases are usually not diagnosed until an advanced stage, leading to a low survival rate of $20-25 \%$.[8-10] It is of great importance to identify novel biomarkers associated with GC pathogenesis to improve the monitoring of the disease progression.

Osteoglycin (OGN) is a member of the small leucine-rich proteoglycans (SLRPs), a family of extracellular proteoglycans with several leucine-rich repeats.[11, 12] As a component of extracellular matrix (ECM), 
OGN can bind collagen as well as several growth factors including transforming growth factor- $\beta$ (TGF- $\beta$ ), [13] epidermal growth factor receptors (EGFRs) and insulin growth factor receptors (IGFs),[14] playing key roles in ECM remodeling and tissue development.[15, 16] Recent studies have shown that downregulation of OGN is involved in the progression and metastasis of various human cancers such as squamous cervical carcinoma, vaginal cancer, colorectal adenoma, and laryngeal carcinoma.[11, 14, 17] In colorectal cancer, for example, by binding EGFRs and facilitating their endocytosis and degradation, OGN plays a tumor suppressive role by mediating the negative feedback control of EGFR signaling.[18] In accordance, lower OGN expression is an indicator of decreased survival and higher cancer recurrence in colorectal cancer.[14, 17, 19] Genome-scale transcriptomic analysis has revealed significant downregulation of OGN expression in GC tissues compared to non-tumorous stomach tissues,[20] however, the relationship between its expression and the clinic-pathological features of GC has not yet been investigated. In this study, we evaluated the expression of OGN in gastric cancer specimens and examine its pathologic significance.

\section{Methods}

\section{Patient samples and Ethics statement}

All of the tissue blocks used in this study were obtained from the General Surgery of the Affiliated Hospital of Jiangnan University from October 2017 to April 2018. There were 44 patients in total and no patient had received preoperative chemotherapy or radiotherapy. Forty adjacent non-tumorous tissues were obtained by gastrectomy $(>5 \mathrm{~cm})$. All of the samples were independently evaluated and confirmed by two pathologists. GC tumor types were classified according to the 2002 World Health Organization (WHO) GC histological classification and Lauren classification.[21] The final stage of the sample was determined according to the Union for International Cancer Control (UICC) classification system. All of the tissue samples were obtained with the patients' written consent, and the research protocol was approved by the Human Research Ethics Committee of the Affiliated Hospital of Jiangnan University.

\section{Immunohistochemistry (IHC) and quantification}

The expression level of OGN protein was determined by IHC. The tissue sections were first dewaxed in xylene and passed through a gradient of ethanol to distilled water. The tissue sections were mixed with sodium citrate antigen retrieval buffer $(\mathrm{pH} \mathrm{8.0)}$ and boiled in a microwave oven for $10 \mathrm{~min}$. After cooling, the tissue sections were treated with $3 \% \mathrm{H}_{2} \mathrm{O}_{2}$ for 10 min to block endogenous peroxidase activity. The tissue sections were then blocked with $2 \%$ bovine serum albumin (catalog number A8020; Solarbio) and incubated with OGN antibody at $4{ }^{\circ} \mathrm{C}$ overnight. An EnVision detection system (Dako) was used to stain the OGN in the tissue microarrays (TMA). After washing them three times with PBS, the sections were incubated with secondary antibodies and stained with 3,3'-diaminobenzidine reagent. The tissue sections were then counterstained with hematoxylin, dehydrated with gradient alcohol, clarified in xylene, and mounted with neutral resin. 


\section{IHC quantification and statistical analysis}

IHC staining was evaluated in a semiquantitative method by scoring the intensity on a scale from 0 to 3 $(0$, negative; 1 , weak or scattered staining in a minority of cells; 2 , moderate or strong staining in a majority of cells; and 3, strong staining in all cells), as reported previously.[22] For the statistical analysis, the patients were stratified into OGNLow (score of 0-1) vs OGNHigh (score of 2-3) groups. The statistical analyses were conducted using GraphPad Prism software version 6.0, and the differences between the expression of OGN and the clinic-pathological variables were examined using the Chi-Squared test and the Student's t-test. $P \leq 0.05$ was considered statistically significant.

\section{Results}

\section{Clinicopathological data}

Among the 44 patients studied, 21 (61\%) were men and 17 (39\%) were women, with a mean age of 65 years (range, 53 to 82 years). Total gastrectomy was performed in 21 patients and distal subtotal gastrectomy in 23 patients. Thirty-six patients had H. pylori infection. The histological examination after surgery showed 5 cases of mucinous type, 29 cases of tubular type, 1 case of signet-ring cell carcinoma, and 9 cases of others. Twenty-five (57\%) of the studied tumors were classified as moderately differentiated type and 19 (43\%) as poorly differentiated type. According to TNM classification, 15 (34\%) were of limited (T1/2) extent and 29 (66\%) were locally advanced (T3/4). By pathological and clinical assessment, the majority of patients had lymphatic metastases (N1-N3; 73\%). In contrast, only 4 cases had distant metastases (M1) at the time of surgery. Twenty-three cases showed presence of lymphatic tumor emboli. Five (11\%) cases had stage I disease, 17 (39\%) had stage II disease, 18 (41\%) cases had stage III disease and 4 (9\%) cases had stage III disease (Table 1). 
Table 1

Patient and tumor characteristics

\begin{tabular}{|ll|}
\hline & Patient characteristics \\
\hline Total number & 44 \\
\hline Age (years) & \\
\hline Median [Range] & $65[53-82]$ \\
\hline Gender & \\
\hline Female & $17(39 \%)$ \\
\hline Male & $27(61 \%)$ \\
\hline T-Status & \\
\hline 1 & $1(2 \%)$ \\
\hline 2 & $14(32 \%)$ \\
\hline 3 & $2(4 \%)$ \\
\hline 4 & $27(62)$ \\
\hline N-Status & \\
\hline 0 & $12(27 \%)$ \\
\hline 1 & $8(18 \%)$ \\
\hline 2 & $17(39 \%)$ \\
\hline 3 & $7(16 \%)$ \\
\hline M-Status & $17(91 \%)$ \\
\hline 0 & \\
\hline 1 & \\
\hline II & \\
\hline I & \\
\hline
\end{tabular}




\begin{tabular}{|lc|}
\hline & Patient characteristics \\
\hline II & $17(39 \%)$ \\
\hline III & $18(41 \%)$ \\
\hline H. pylori infection & $4(9 \%)$ \\
\hline+ & $36(82 \%)$ \\
\hline- & $8(18 \%)$ \\
\hline Tumor emboli & $23(52 \%)$ \\
\hline+ & $21(48 \%)$ \\
\hline- & $14(32 \%)$ \\
\hline Histologic differentiation \\
\hline Moderated & $30(68 \%)$ \\
\hline Poorly & \\
\hline
\end{tabular}

Expression of OGN decreased in the GC tissues compared with the adjacent normal tissues

To determine whether the expression of OGN was dysregulated in gastric cancer, IHC was performed on the TMA with $44 \mathrm{GC}$ tissues and 40 non-tumorous stomach tissues. OGN expression was seen throughout the foveolar and glandular epithelium of the mucosa $(38 / 40,95 \%)$, with the basal segments of the gland showing more intensive staining (Fig. 1A). In contrast, GC samples revealed varying expression intensities of OGN ranging from strong to absent (Fig. 1A). Quantitative analysis of the IHC results, as shown in (Fig. 1B), showed that loss of OGN expression occurred in $75 \%$ of gastric carcinoma samples $(P<0.001)$. These results indicate that the expression of $O G N$ in the $G C$ tissues was significantly reduced than that in the non-tumorous tissues.

\section{Ogn Expression Correlates With Histological Differentiation Of Gc}

The degree of tumor differentiation is an important indicator of tumor malignancy, with poorly differentiated tumors tending to be more aggressive.[23] To study whether the expression level of OGN protein is related to the degree of GC differentiation, we compared the expression of OGN in GC tissues with different differentiation status (Fig. 2A). The statistical analysis showed that expression levels of OGN in the poorly differentiated GC tissues were significantly lower than those in the moderately differentiated GC tissues ( $P=0.0015$, Fig. $2 B$ ). This result indicates $O G N$ expression in GC is negatively correlated with the degree of tumor differentiation. 
GC samples with lower OGN expression demonstrated higher degree of lymph node metastasis

We further examined the association of OGN expression levels with several other clinic-pathological parameters of the GC patients. There was no significant correlation between the levels of OGN and the gastric cancer subtype, Borrmann classification, status of tumor emboli, histological type, or H. pylori infection status (Table 2). 
Table 2

Association of expression of OGN with clinicopathological characteristics in gastric cancer patients

\begin{tabular}{|c|c|c|c|}
\hline \multirow[t]{2}{*}{ Characteristic } & \multicolumn{3}{|l|}{ OGN } \\
\hline & High expression & Low expression & $p$ \\
\hline \multicolumn{4}{|l|}{ Total } \\
\hline Cancer & $11(25 \%)$ & 33 (75\%) & \multirow[t]{2}{*}{0.000} \\
\hline Adjacent normal tissue & $38(95 \%)$ & $2(5 \%)$ & \\
\hline \multicolumn{4}{|l|}{ Histologic differentiation } \\
\hline medium & $8(57 \%)$ & $6(43 \%)$ & \multirow[t]{2}{*}{0.001} \\
\hline Poorly & $3(10 \%)$ & $27(90 \%)$ & \\
\hline \multicolumn{4}{|l|}{ N-status } \\
\hline $0 \& 1$ & $8(40 \%)$ & $12(60 \%)$ & \multirow[t]{2}{*}{0.036} \\
\hline $2 \& 3$ & $3(13 \%)$ & $21(87 \%)$ & \\
\hline \multicolumn{4}{|l|}{ Borrmann classification } \\
\hline I & $3(43 \%)$ & $4(57 \%)$ & \multirow[t]{4}{*}{0.250} \\
\hline II & $2(12 \%)$ & 15 (88\%) & \\
\hline III & $6(33 \%)$ & $12(67 \%)$ & \\
\hline IV & $0(0 \%)$ & $2(100 \%)$ & \\
\hline \multicolumn{4}{|l|}{ Stage } \\
\hline I & $1(20 \%)$ & $4(80 \%)$ & \multirow[t]{4}{*}{0.218} \\
\hline II & $7(41 \%)$ & $10(59 \%)$ & \\
\hline III & $3(17 \%)$ & 15 (83\%) & \\
\hline IV & $0(0 \%)$ & $4(100 \%)$ & \\
\hline \multicolumn{4}{|l|}{ H. pylori infection } \\
\hline+ & $6(75 \%)$ & $2(25 \%)$ & \multirow[t]{2}{*}{0.549} \\
\hline- & $23(64 \%)$ & $13(36 \%)$ & \\
\hline \multicolumn{4}{|l|}{ Tumor emboli } \\
\hline+ & $14(61 \%)$ & $9(39 \%)$ & \multirow[t]{2}{*}{0.461} \\
\hline- & $15(71 \%)$ & $6(29 \%)$ & \\
\hline
\end{tabular}




\begin{tabular}{|c|c|c|c|}
\hline \multirow[t]{2}{*}{ Characteristic } & \multicolumn{3}{|l|}{ OGN } \\
\hline & High expression & Low expression & $p$ \\
\hline \multicolumn{4}{|c|}{ Gastric carcinoma subtype } \\
\hline Intestinal & $5(19 \%)$ & $22(81 \%)$ & \multirow[t]{3}{*}{0.324} \\
\hline Diffuse & $1(14 \%)$ & $6(86 \%)$ & \\
\hline Mixed & $4(40 \%)$ & $6(60 \%)$ & \\
\hline \multicolumn{4}{|c|}{ Histological type } \\
\hline Tubular & $6(21 \%)$ & $23(79 \%)$ & \multirow[t]{4}{*}{0.670} \\
\hline Mucinous & $2(40 \%)$ & $3(60 \%)$ & \\
\hline Signet ring & $0(0 \%)$ & $1(100 \%)$ & \\
\hline Others & $3(33 \%)$ & $6(67 \%)$ & \\
\hline
\end{tabular}

Tumor invasion and metastasis is one of the main causes of death in patients with GC.[24, 25] Notably, GC patients with lower OGN expression were more prone to exhibit higher degree of lymph node metastasis than patients with higher OGN expression (Fig. 3A and 3B). These results indicate that decreased OGN expression is associated with enhanced lymph node metastasis in GC.

\section{Discussion}

GC is one of the leading causes of cancer-related death with limited therapeutic options.[26] Identification of disease biomarkers and drug targets is urgently needed. In the present study, we analyzed OGN expression levels in a cohort of gastric cancer patients and correlated to their clinico-pathological features. Our results showed that the expression levels of OGN in gastric cancer tissues were significantly reduced compared to the normal counterparts. In addition, lower OGN expression was associated with higher lymph node metastasis and poor differentiation status, which are indicators of more advanced malignancy. Together, these results demonstrated that OGN downregulation might participate in the progression of gastric cancer, and suggested OGN as a potential biomarker for cancer diagnosis and monitoring in GC.

Several studies have shown that OGN is downregulated in a varity of human cancers including squamous cervical carcinoma, colorectal adenoma and breast cancer, suggesting that it may function as a tumor suppressor. For example, OGN was shown to reduce EGFR/Akt/Zeb-1 activity by enhancing EGFR dimerization and endocytosis, leading to inhibition of epithelial-mesenchymal transition, reduced cell proliferation and invasion in colon cancer cells. $[18,19]$ Notably, recent study by Hu et al. demonstrated an immuno-regulatory role of OGN in cancer progression, by which OGN attenuates HIF-1a/VEGF signaling axis and thus inhibits CD8 + cell infiltration. $[14,27]$ Our observation that GC patients with low OGN expression were associated with higher lymph node metastasis and lower differentiation status is in 
accordance with these findings. As epithelial-mesenchymal transition (EMT) and evasion of immune destruction are typical features of advanced malignancies,[28-30] further research should be conducted to investigate the mechanism by which OGN may be involved in these biological processes.

\section{Conclusions}

Collectively, this study identified that OGN expression was significantly decreased in gastric cancer tissues. Furthermore, the result indicated that reduced OGN expression was related to higher lymph node metastasis and poorly differentiation status. It is necessary to further study the molecular mechanism of OGN in the initiation and development of gastric cancer, which may give insights into the molecular basis of the role of OGN in GC progression and metastasis, and provide potential novel therapeutic target for GC treatment.

\section{Abbreviations}

\begin{tabular}{|ll|}
\hline GC & Gastric cancer \\
\hline OGN & Osteoglycin \\
\hline IHC & Immunohistochemistry \\
SLRPs & Small leucine-rich proteoglycans \\
\hline ECM & Extracellular matrix \\
\hline TGF- $\beta$ & Transforming growth factor- $\beta$ \\
\hline EGFRs & Epidermal growth factor receptors \\
\hline IGFs & Insulin growth factor receptors \\
\hline WHO & World health organization \\
\hline UICC & Union for International Cancer Control \\
\hline TMA & Tissue microarrays \\
\hline PBS & Phosphate-buffered saline \\
\hline ZEB1 & Zinc finger E-box binding homeobox 1 \\
\hline HIF-1a & Hypoxia inducible factor 1 subunit alpha \\
\hline VEGF & Vascular endothelial growth factor \\
\hline EMT & Epithelial-mesenchymal transition \\
\hline
\end{tabular}

\section{Declarations}


The research protocol was approved by the Human Research Ethics Committee of the Affiliated Hospital of Jiangnan University (LS 2017091), and all of the tissue samples were obtained with the patients' written consent.

\section{Consent to publish}

Not applicable.

\section{Availability of data and materials}

The datasets used or analysed during the current study are available from the corresponding author on reasonable request.

\section{Competing interests}

None of the authors has a conflict of interest to disclose.

\section{Funding}

This work was supported by the Jiangnan University Public Health Research Center Foundation for Youths to Chengjia Qian (JUPH201827) and the Academician Li Zhaoshen Workstation of Jiangnan University Affiliated Hospital to Huiming Tu (C2019301).

\section{Authors' Contributions}

$\mathrm{BF}$ and $\mathrm{HT}$ conceived and designed the experiments. CQ and CZ performed the experiments. CQ analyzed the data. QG, JG, WH, HJ, LJ, XQ, BF and HT contributed the reagents/materials/ analysis tools. CQ and $\mathrm{CZ}$ wrote the manuscript. All authors read and approved the final manuscript.

\section{Acknowledgements}

We gratefully acknowledge the cooperation of each researcher and the great support given by the Affiliated Hospital of Jiangnan University.

\section{References}

1. Tan P, Yeoh KG. Genetics and Molecular Pathogenesis of Gastric Adenocarcinoma. Gastroenterology. 2015;149(5):1153-62.e3.

2. Huang XM, Liu XS, Lin XK, Yu H, Sun JY, Liu XK, Chen C, Jin HL, Zhang GE, Shi XX, Zhang Q, Yu JR. Role of plasmacytoid dendritic cells and inducible costimulator-positive regulatory $T$ cells in the immunosuppression microenvironment of gastric cancer. Cancer Sci. 2014;105(2):150-8.

3. Liu X, Yu H, Yan C, Mei Y, Lin C, Hong Y, Lin X, Zhang Q, Yu J. Plasmacytoid Dendritic Cells and ICOS(+) Regulatory T Cells Predict Poor Prognosis in Gastric Cancer: A Pilot Study. J Cancer. 2019;10(26):6711-5. 
4. Zhu L, Chen W, Li G, Chen H, Liao W, Zhang L, Xiao X. Upregulated RACK1 attenuates gastric cancer cell growth and epithelial-mesenchymal transition via suppressing Wnt/ $\beta$-catenin signaling. OncoTargets therapy. 2019;12:4795-805.

5. Xue F, An C, Chen L, Liu G, Ren F, Guo X, Sun H, Mei L, Sun X, Li J, Tang Y, An X, Zheng P. 4.1B suppresses cancer cell proliferation by binding to EGFR P13 region of intracellular juxtamembrane segment. Cell communication signaling: CCS. 2019;17(1):115.

6. Polkowska-Pruszyńska B, Rawicz-Pruszyński K, Ciseł B, Sitarz R, Polkowska G, Krupski W, Polkowski WP. Liver metastases from gastric carcinoma: A Case report and review of the literature. Curr Probl Cancer. 2017;41(3):222-30.

7. Min K, Lee SK. EBV miR-BART10-3p Promotes Cell Proliferation and Migration by Targeting DKK1. Int J Biol Sci. 2019;15(3):657-67.

8. Qu CY, Zhou M, Chen YW, Chen MM, Shen F, Xu LM. Engineering of lipid prodrug-based, hyaluronic acid-decorated nanostructured lipid carriers platform for 5 -fluorouracil and cisplatin combination gastric cancer therapy. Int J Nanomed. 2015;10:3911-20.

9. Laboissiere RS, Buzelin MA, Balabram D, De Brot M, Nunes CB, Rocha RM, Cabral MM, Gobbi H. Association between HER2 status in gastric cancer and clinicopathological features: a retrospective study using whole-tissue sections. BMC Gastroenterol. 2015;15:157.

10. Lv D, Xing C, Cao L, Zhuo Y, Wu T, Gao N. PD-L1 gene promoter methylation represents a potential diagnostic marker in advanced gastric cancer. Oncology letters. 2020;19(2):1223-34.

11. Li L, Zhang Z, Wang C, Miao L, Zhang J, Wang J, Jiao B, Zhao S. Quantitative proteomics approach to screening of potential diagnostic and therapeutic targets for laryngeal carcinoma. PloS one. 2014;9(2):e90181.

12. Serafín-Higuera I, Garibay-Cerdenares OL, Illades-Aguiar B, Flores-Alfaro E, Jiménez-López MA, SierraMartínez P. C. Alarcón-Romero Ldel, Differential proteins among normal cervix cells and cervical cancer cells with HPV-16 infection, through mass spectrometry-based Proteomics (2D-DIGE) in women from Southern México. Proteome science. 2016;14(1):10.

13. Yano K, Washio K, Tsumanuma Y, Yamato M, Ohta K, Okano T, Izumi Y. The role of Tsukushi (TSK), a small leucine-rich repeat proteoglycan, in bone growth. Regenerative therapy. 2017;7:98-107.

14. Hu X, Li YQ, Li QG, Ma YL, Peng JJ, Cai SJ. Osteoglycin-induced VEGF Inhibition Enhances T Lymphocytes Infiltrating in Colorectal Cancer. EBioMedicine. 2018;34:35-45.

15. Andenæs K, Lunde IG, Mohammadzadeh N, DahI CP, Aronsen JM, Strand ME, Palmero S, Sjaastad I, Christensen G, Engebretsen KVT, Tønnessen T. The extracellular matrix proteoglycan fibromodulin is upregulated in clinical and experimental heart failure and affects cardiac remodeling. PloS one. 2018;13(7):e0201422.

16. Seher A, Nickel J, Mueller TD, Kneitz S, Gebhardt S, ter Vehn TM, Schlunck G, Sebald W. Gene expression profiling of connective tissue growth factor (CTGF) stimulated primary human tenon fibroblasts reveals an inflammatory and wound healing response in vitro. Molecular vision. 2011;17:53-62. 
17. Xu T, Zhang R, Dong M, Zhang Z, Li H, Zhan C, Li X, Osteoglycin (OGN) Inhibits Cell Proliferation and Invasiveness in Breast Cancer via PI3K/Akt/mTOR Signaling Pathway, OncoTargets and therapy 12 (2019) 10639-10650.

18. Hu X, Li YQ, Li QG, Ma YL, Peng JJ, Cai SJ. Osteoglycin (OGN) reverses epithelial to mesenchymal transition and invasiveness in colorectal cancer via EGFR/Akt pathway. Journal of experimental clinical cancer research: CR. 2018;37(1):41.

19. Dimberg A. Osteoglycin - A switch from angiogenesis to T-cell recruitment? EBioMedicine. 2018;35:22-3.

20. Lee JY, Eom EM, Kim DS, Ha-Lee YM, Lee DH. Analysis of gene expression profiles of gastric normal and cancer tissues by SAGE. Genomics. 2003;82(1):78-85.

21. Liu X, Chu KM. E-cadherin and gastric cancer: cause, consequence, and applications. BioMed research international. 2014;2014:637308.

22. Li X, Yang J, Sciences JLJAoB. Liprin- $\beta 1$ is up regulated in human hepatocellular carcinoma and is associated with advanced tumor stage, 71 (2019) 29-29.

23. Ducarouge B, Pelissier-Rota M, Lainé M, Cristina N, Vachez Y, Scoazec JY, Bonaz B, Jacquier-Sarlin M. CRF2 signaling is a novel regulator of cellular adhesion and migration in colorectal cancer cells. PloS one. 2013;8(11):e79335.

24. Yao HH, Wang BJ, Wu Y, Huang Q. High Expression of Angiogenic Factor with G-Patch and FHA Domain1 (AGGF1) Predicts Poor Prognosis in Gastric Cancer. Medical science monitor: international medical journal of experimental clinical research. 2017;23:1286-94.

25. Lai H, Jin Q, Lin Y, Mo X, Li B, He K, Chen J. Combined use of lysyl oxidase, carcino-embryonic antigen, and carbohydrate antigens improves the sensitivity of biomarkers in predicting lymph node metastasis and peritoneal metastasis in gastric cancer. Tumour biology: the journal of the International Society for Oncodevelopmental Biology Medicine. 2014;35(10):10547-54.

26. Zhang LH, Wang Z, Li LH, Liu YK, Jin LF, Qi XW, Zhang C, Wang T, Hua D. Vestigial like family member 3 is a novel prognostic biomarker for gastric cancer. World journal of clinical cases. 2019;7(15):1954-63.

27. Wu QH, Ma Y, Ruan CC, Yang Y, Liu XH, Ge Q, Kong LR, Zhang JW, Yan C, Gao PJ. Loss of osteoglycin promotes angiogenesis in limb ischaemia mouse models via modulation of vascular endothelial growth factor and vascular endothelial growth factor receptor 2 signalling pathway. Cardiovascular research. 2017;113(1):70-80.

28. Hanahan D, Weinberg RA. Hallmarks of cancer: the next generation. Cell. 2011;144(5):646-74.

29. Zhang P, Lu X, Shi Z, Li X, Zhang Y, Zhao S, Liu H. miR-205-5p regulates epithelial-mesenchymal transition by targeting PTEN via PI3K/AKT signaling pathway in cisplatin-resistant nasopharyngeal carcinoma cells. Gene. 2019;710:103-13.

30. Jiang Y, Li W, Lu J, Zhao X, Li L. HLA-G + 3142 C > G polymorphism and cancer risk: Evidence from a meta-analysis and trial sequential analysis. Medicine. 2019;98(25):e16067. 
Figures

A
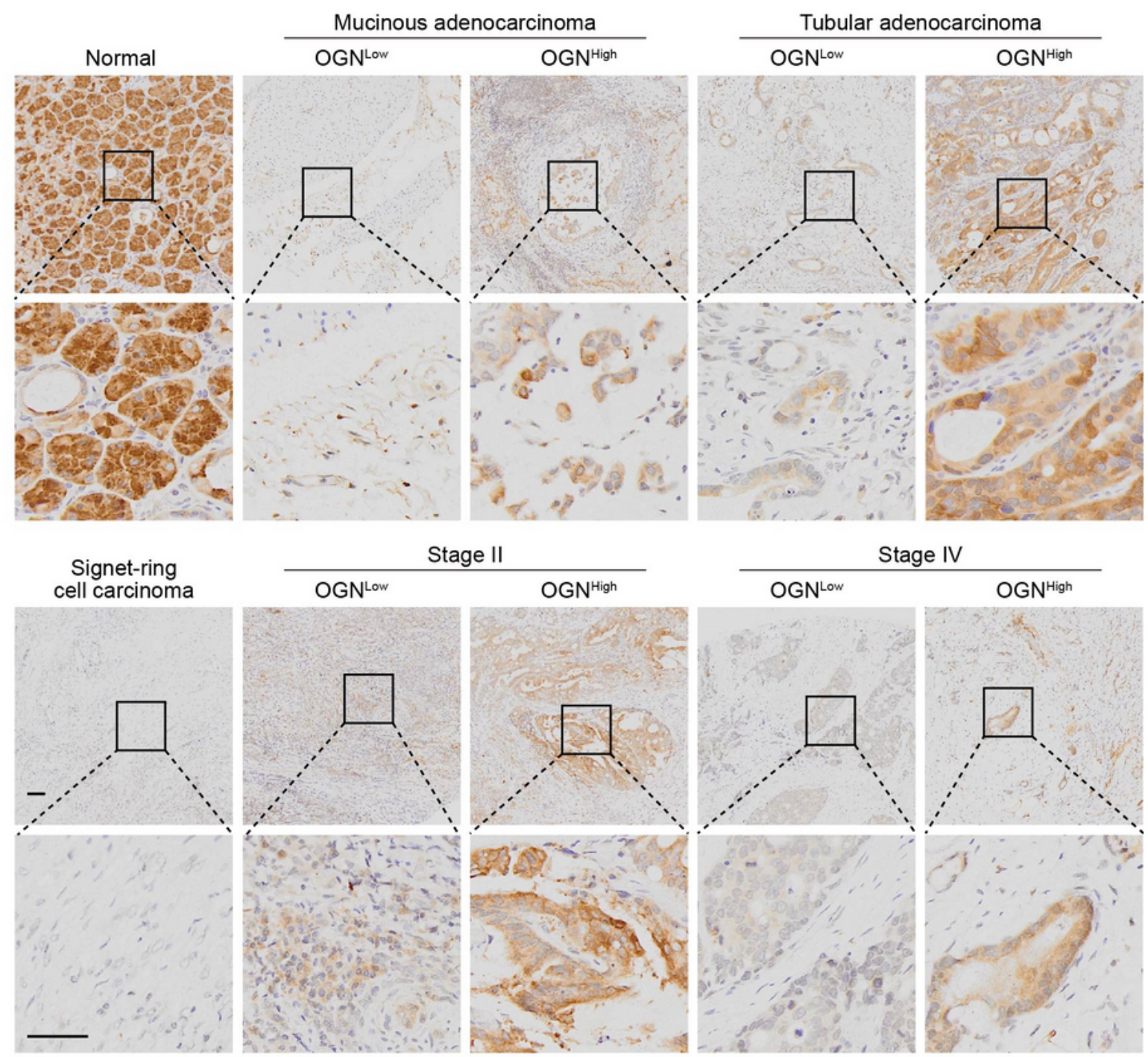

B

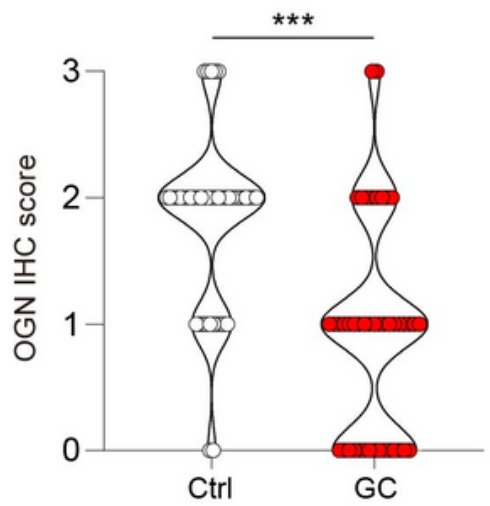

Figure 1

OGN expression in gastric cancer tissues and adjacent normal tissues. 
A. IHC of OGN in gastric cancer tissues and adjacent normal tissues. Tumor samples were layered into $\mathrm{OGN}^{\mathrm{LOW}}$ and $\mathrm{OGN} \mathrm{High}^{\mathrm{Hig}}$ groups and representative images are displayed. Scale bar, $50 \mu \mathrm{m}$. B. Quantification of OGN staining in gastric cancer tissues and adjacent normal tissues $(P<0.001)$.

A

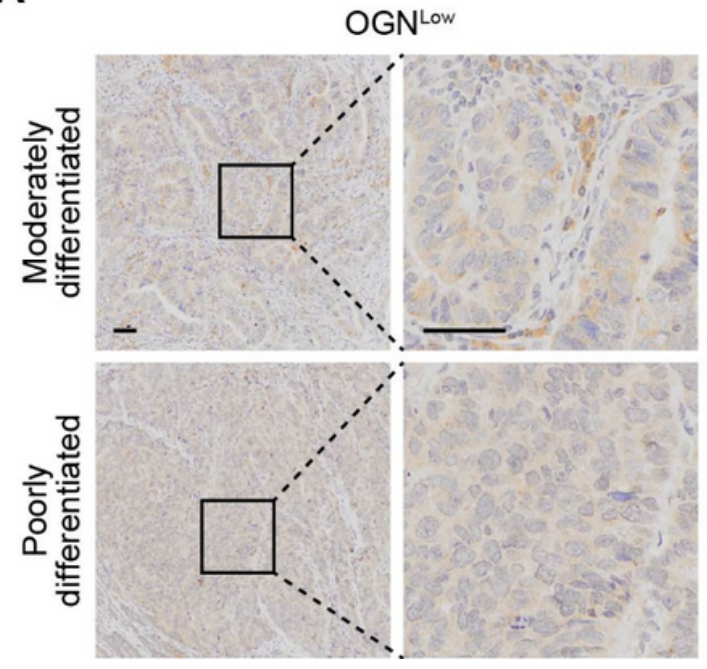

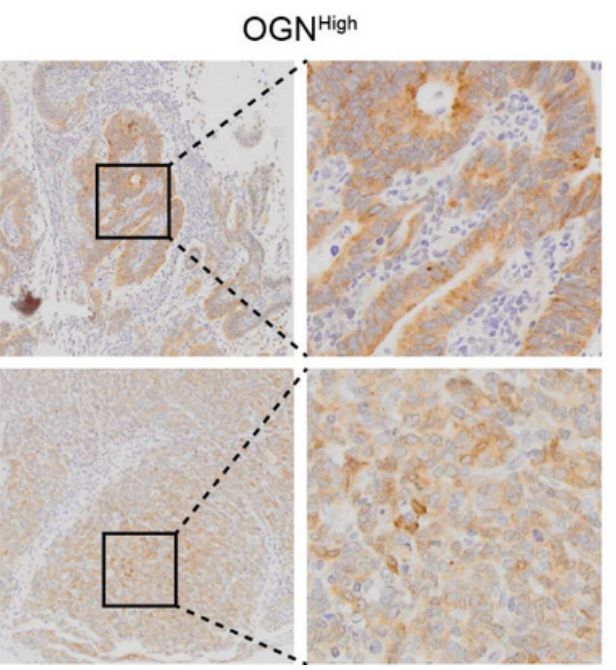

B

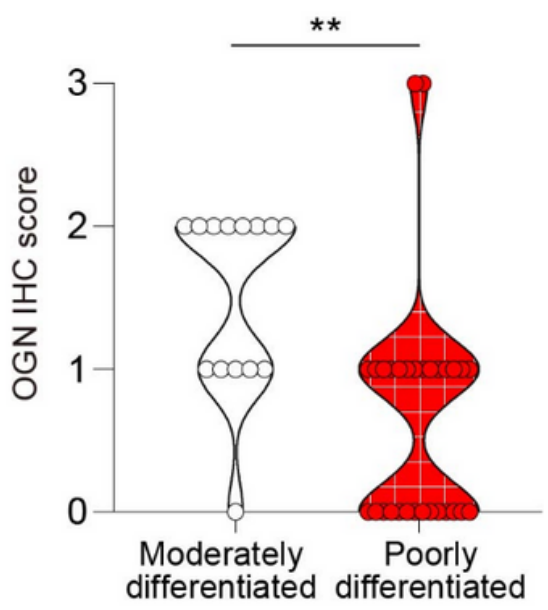

Figure 2

OGN expression in medium differentiation and poorly differentiated tissues in gastric cancer.

A. IHC of OGN in medium differentiation and poorly differentiated tissues of gastric cancer. Tumor samples were layered into $O G N^{\mathrm{LOW}}$ and $O G N^{\text {High }}$ groups and representative images are displayed. Scale bar, $50 \mu \mathrm{m}$. B. Quantification of OGN staining in medium differentiation and poorly differentiated tissues of gastric cancer $(P<0.01)$.

A

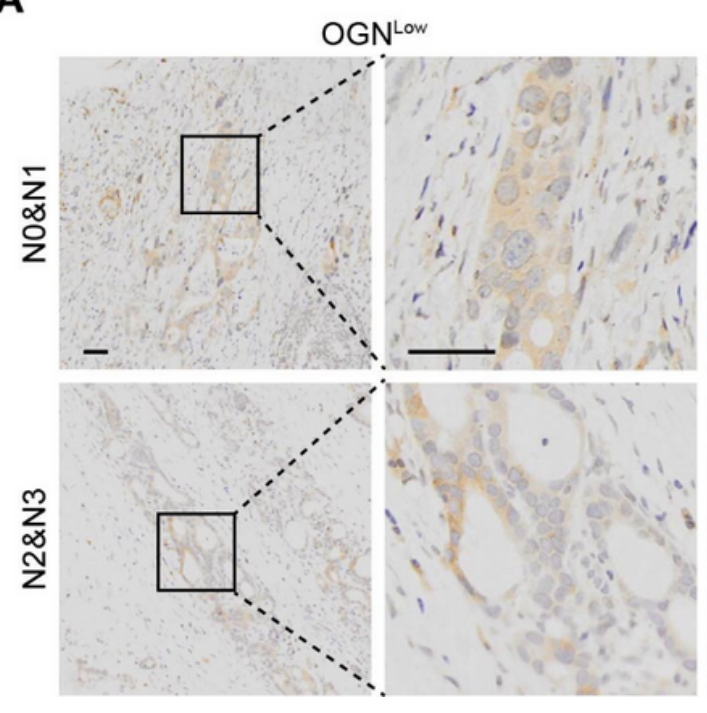

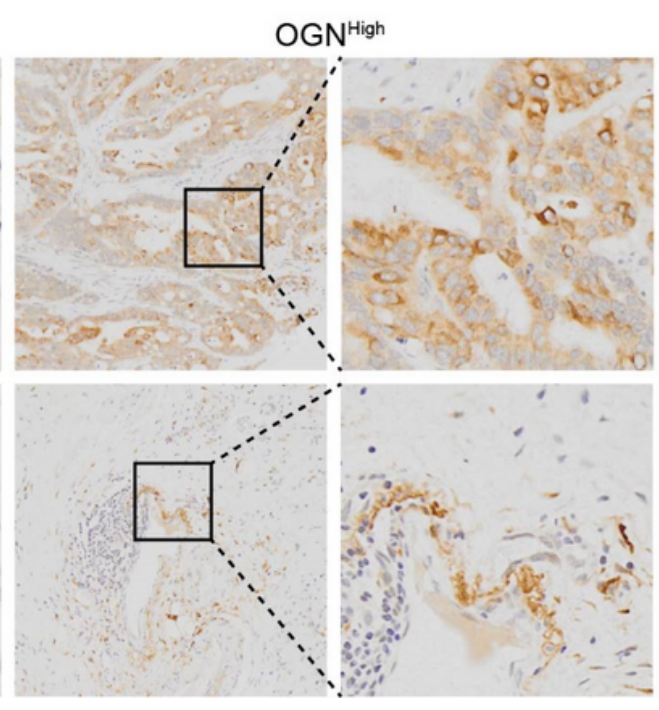

B

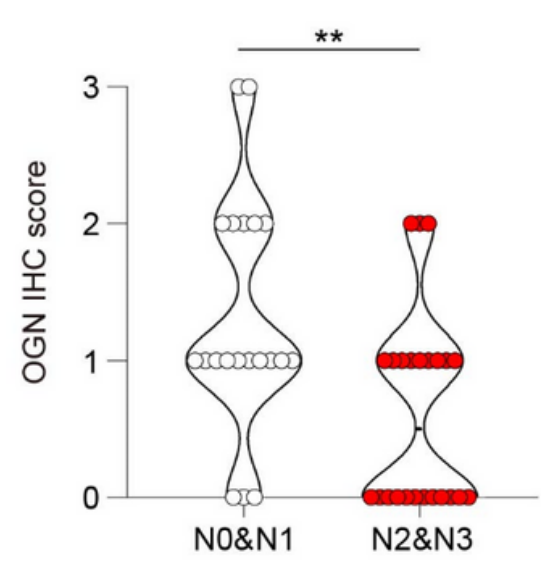

Figure 3

OGN expression in different degrees of lymph node metastasis. 
A. IHC of OGN in gastric cancer with different degrees of lymph node metastasis. Tumor samples are layered into $O G N^{\text {Low }}$ and $O G N^{\text {High }}$ groups and representative images are displayed. Scale bar, $50 \mu \mathrm{m}$. B. Quantification of OGN staining in different degrees of lymph node metastasis in gastric cancer $(P<0.01)$. 